Revue internationale P.M.E.

Économie et gestion de la petite et moyenne entreprise

\title{
Sur les traces des créateurs: vers un modèle de comportement entrepreneurial
}

\section{Émile-Michel Hernandez}

Volume 7, numéro 1, 1994

URI : https://id.erudit.org/iderudit/1008372ar

DOI : https://doi.org/10.7202/1008372ar

Aller au sommaire du numéro

Éditeur(s)

Presses de l’Université du Québec

ISSN

0776-5436 (imprimé)

1918-9699 (numérique)

Découvrir la revue

Citer cette note

Hernandez, É.-M. (1994). Sur les traces des créateurs: vers un modèle de comportement entrepreneurial. Revue internationale P.M.E., 7(1), 127-139. https://doi.org/10.7202/1008372ar
Résumé de l'article

L'activité du créateur d'entreprise peut-elle être analysée comme une variante de celle du cadre ou bien est-elle d'une tout autre nature ? Pour répondre à cette question, une recherche, comparable à celle de Mintzberg sur cinq cadres, a été réalisée sur cinq créateurs. Cette étude comporte deux parties : la première porte sur les rôles professionnels présentés par Mintzberg; la seconde, sur une variable de contingence : la taille de l'organisation. 


\title{
Note de recherche
}

\section{Sur les traces des créateurs: vers un modèle de comportement entrepreneurial}

\author{
Émile-Michel HERNANDEZ* \\ Maître de conférences en sciences de gestion
}

\begin{abstract}
RÉSUMÉ
L'activité du créateur d'entreprise peut-elle être analysée comme une variante de celle du cadre ou bien est-elle d'une tout autre nature? Pour répondre à cette question, une recherche, comparable à celle de Mintzberg sur cinq cadres, a été réalisée sur cinq créateurs. Cette étude comporte deux parties : la première porte sur les rôles professionnels présentés par Mintzberg; la seconde, sur une variable de contingence: la taille de l'organisation.
\end{abstract}

\section{ABSTRACT}

Can the activity of an entrepreneur be analyzed as a variant of that of a manager, or is it totally different? To answer this question, research comparable to Mintzberg's work on five managers was conducted among five entrepreneurs. This study includes two parts: the first deals with the professional roles presented by Mintzberg; the second focuses on a contingent variable, the size of the organization.

\section{RESUMEN}

Puede la actividad de un creador de empresa ser analizada como una variante de la de un ejecutivo o bien tiene otro matiz? Para contestar a esta pregunta, una investigación, que se puede comparar a la que llevó a cabo Mintzberg sobre cinco ejecutivos, se realizó sobre cinco creadores. Este estudio consta de dos partes, la primera trata de los papeles profesionales presentados por Mintzberg, la segunda de una variable contingente: el tamaño de la organización.

* Émile Hernandez est maître de conférences en sciences de gestion. Adresse : 4, Allée des Albatros, 44600 Saint-Nazaire, France. 


\section{Introduction}

L'étude de l'entrepreneuriat constitue un domaine très vaste qui n'a été que partiellement exploré par les chercheurs. Un des thèmes les plus étudiés est celui des acteurs, c'est-à-dire des personnes qui sont à l'origine des créations d'entreprise. De nombreux travaux, essentiellement aux États-Unis, ont été consacrés à l'examen de leurs caractéristiques psychologiques et sociologiques. Ce thème est encore présent dans les préoccupations des chercheurs. Pourtant, il subit actuellement une désaffection certaine. Ces études n'ont pas permis de dresser un profil sociologique ou psychologique capable de distinguer ceux qui sont les plus susceptibles de créer une entreprise des autres; et elles n'ont pas permis non plus de distinguer clairement le profil psychologique de ceux qui réussissent de ceux qui échouent. Le deuxième aspect de l'étude des acteurs a porté sur leurs motivations à créer une entreprise. Tous ces travaux présentent la caractéristique commune de relever d'une approche qualifiée par William B. Gartner (1989) d' «approche caractérielle». Et dans un article intitulé «Who is an entrepreneur? Is the wrong question », cet auteur se demande si la relative improductivité de ces recherches ne devrait pas amener maintenant les chercheurs à ne plus se demander «Qui est le créateur?» mais «Que fait le créateur?» Il qualifie cette nouvelle approche de comportementale: «La recherche sur l'entrepreneur devrait se focaliser sur ce qu'il fait et non sur ce qu'il est». Cet auteur, Gartner et un autre (Bird, 1986) citent Mintzberg et les travaux qu'il a menés sur le comportement des managers pour appuyer leurs propos. Gartner propose d'utiliser la même méthode pour l'entrepreneuriat: «La recherche sur l'entrepreneuriat devrait suivre le même chemin que la recherche dans le domaine des comportements des dirigeants... Je crois que la recherche sur les comportements des entrepreneurs doit être fondée sur un travail de terrain semblable à l'étude de Mintzberg sur le travail des dirigeants. Les chercheurs doivent observer les entrepreneurs lorsqu'ils sont en train de créer des organisations. Ce travail doit être décrit en détail et les activités systématisées et classifiées. La connaissance des comportements des créateurs dépend du travail sur le terrain. » Nous avons donc suivi ses conseils et mené auprès des créateurs d'entreprise une recherche comparable à celle de Mintzberg (1984) sur les managers.

Il s'est demandé en quoi consistait réellement leur travail. Pour répondre à cette question, il a passé 5 jours avec 5 directeurs généraux, soit 25 jours d'observation, à étudier le comportement réel de ces managers. Et il s'est aperçu que la réalité était assez différente de la conception classique du travail du cadre telle que la présentent des auteurs comme Fayol ou Luther Gulick. À la suite de ses observations directes, Mintzberg a relevé que le travail du manager présente six caractéristiques essentielles : beaucoup de travail et un rythme soutenu; une activité caractérisée par la brièveté, la variété et la fragmentation; la préférence pour l'action; l'importance de la communication verbale; le cadre est la 
charnière entre son organisation et un réseau de contacts; un mélange de droits et de devoirs. Ce travail peut être décomposé en dix rôles professionnels: trois rôles interpersonnels (symbole, leader et agent de liaison), trois rôles liés à l'information (observateur actif, diffuseur et porte-parole) et quatre rôles décisionnels (entrepreneur, régulateur, répartiteur de ressources et négociateur). Enfin, observant la diversité des postes de cadre, il propose une théorie contingente de leur travail.

La recherche menée sur les créateurs utilise la même méthode: l'observation directe. Mais au-delà de cette méthode commune, les résultats s'appuient sur ceux obtenus par Mintzberg. Le travail du créateur est présenté en se référant à celui du manager: le créateur n'est-il pas le manager d'une organisation en cours de constitution? Son activité est-elle une variante de celle du cadre ou est-elle d'une tout autre nature?

Avant de présenter les résultats obtenus, il convient d'apporter quelques précisions sur les conditions de réalisation de l'étude. Elle a porté sur cinq créateurs à raison de deux journées entières d'observation par créateur à environ un mois d'intervalle l'une de l'autre, soit en tout dix journées. Il s'agit d'entrepreneurs qui sont tout au début de leur processus de création, puisque les observations ont eu lieu essentiellement moins de trois mois après l'obtention de leur Registre de Commerce et leur installation dans une pépinière d'entreprises.

Cet article comporte deux parties principales. La première porte sur les rôles professionnels présentés par Mintzberg, la seconde sur une variable de contingence: la taille de l'organisation, en se référant d'abord aux travaux de Choran (1969), puis à ceux de Jean-Luc Delpeuch et Anne Lauvergeon (1986). Enfin, après avoir présenté la journée type du créateur, nous analysons certains aspects méthodologiques de la recherche en entrepreneuriat.

\section{Rôles professionnels}

Mintzberg dans son étude a décrit le travail du cadre à l'aide de dix rôles professionnels de base, dont trois interpersonnels, trois liés à l'information et quatre décisionnels. Le modèle qu'il propose est suffisamment général pour permettre une utilisation très large. Il n'envisage pas le cas du créateur d'entreprise, lequel n'entrait pas dans ses préoccupations. Ce sera fait maintenant. Pour chaque rôle, la signification donnée par Mintzberg sera brièvement rappelée, puis nous verrons s'il existe pour le créateur et l'importance qu'il a: anecdotique, comparable en importance au cas du cadre, ou essentiel. Enfin, nous verrons s'il convient ou non de compléter cette liste par de nouveaux rôles qui seraient, eux, spécifiques au créateur. 
Avant d'entrer dans le détail de ces dix rôles, rappelons que pour Sarbin et Allen (1968) un rôle est «un ensemble organisé de comportements appartenant à un poste de travail ou à une position identifiable».

\subsection{Les rôles interpersonnels}

Ces rôles correspondent à des activités ayant en commun d'être directement liées au statut et à l'autorité du cadre, et d'avoir pour conséquence essentielle le développement de relations interpersonnelles.

Le cadre comme symbole. Il doit remplir un certain nombre de devoirs routiniers de nature légale ou sociale, inhérents à l'autorité formelle dont il est investi. Ainsi, il assiste à des cérémonies, répond à des demandes qui lui sont adressées en raison de son statut ou à des sollicitations diverses. Le créateur, comme le cadre, symbolise son organisation et la représente; plus même, il «est» l'organisation. Mais son entreprise est encore peu connue et il est rarement sollicité pour la représenter à la différence du cadre dirigeant d'une grande entreprise. Aussi, sur le plan pratique, ce rôle a-t-il pour le créateur une importance réduite.

Le cadre comme leader. C'est un aspect du travail du cadre qui, avec la prise de décision, a donné lieu à une littérature abondante. Ce rôle consiste à assurer la responsabilité de la motivation et de l'activation des surbordonnés, des affectations, de la formation... c'est-à-dire pratiquement toutes les activités du cadre impliquant des subordonnés. L'objectif essentiel du rôle du leader est l'intégration des besoins des individus aux buts de l'organisation. Pour le créateur, ce rôle est bien évidemment d'autant plus réduit que le nombre de subordonnés est faible ( 3 créateurs étudiés sur 5 n'avaient aucun employé). Il prend de l'importance lorsque les effectifs de l'entreprise augmentent, sinon il reste marginal.

Le cadre comme agent de liaison. Ce rôle consiste pour le cadre à relier son organisation à l'environnement. Il s'appuie sur son autorité et son statut pour développer un système de relations externes et pour améliorer la position et le niveau d'information de son unité. Il participe donc à de nombreuses activités impliquant des personnes extérieures à son organisation. Ce rôle est important pour le créateur, car l'intégration dans un réseau relationnel constitue un facteur clé de réussite tel qu'indiqué précédemment. C'est plus difficile pour le créateur que pour le cadre, car ce dernier bénéficie souvent de la réputation de son entreprise. Et ce sera encore plus difficile, si le créateur change de domaine d'activité ou de secteur géographique. De plus, les contacts des créateurs, essentiellement avec des clients et des fournisseurs, sont moins riches et moins variés que ceux des cadres. L'implantation dans une pépinière peut faciliter ce rôle, 
d'une part, en conférant une certaine crédibilité au créateur et, d'autre part, en le faisant bénéficier du réseau relationnel propre à la pépinière.

\subsection{Les rôles liés à l'information}

Ils correspondent à des activités liées à la réception et à la transmission d'informations. Le cadre est le centre nerveux de son organisation. Son accès privilégié à l'information externe et son accès total à l'information interne lui confèrent une position centrale dans la circulation des informations non routinières. Ces rôles sont au nombre de trois.

Le cadre comme observateur actif. Ce rôle consiste à chercher et à recevoir une grande variété d'informations pour développer une compréhension approfondie de l'organisation et de son environnement socio-économique. Le cadre est le centre nerveux de l'information interne et externe de l'organisation. Pour développer ce rôle, le cadre s'occupe du courrier et des contacts divers ayant principalement trait à l'information. Il est essentiel d'acquérir une bonne compréhension des tendances de l'environnement, de s'informer des idées nouvelles, de savoir «saisir l'air du temps». Cette veille permanente permet de détecter les opportunités, d'obtenir les informations qui sont nécessaires pour développer le rôle d'entrepreneur. Pour être efficace, ce rôle d'observateur actif nécessite de disposer d'une grande variété de sources d'information. Le créateur ne devra donc pas se contenter de contacts avec des clients et des fournisseurs, mais il lui faudra constituer un réseau informationnel riche et varié. Enfin, l'information réellement utile a plus souvent une forme orale (rumeur, bouche à oreille, etc.) que l'aspect formel d'un rapport officiel émanant d'un organisme professionnel ou autre. Une fois cette information obtenue, il va s'agir de la diffuser soit à l'intérieur de l'organisation (rôle de diffuseur), soit à l'extérieur (rôle de porte-parole).

Le cadre comme diffuseur. Grâce à la qualité de son accès à l'information, le cadre transmet des données de l'extérieur vers l'intérieur de son organisation, et entre subordonnés. L'information transmise est de deux natures : elle concerne soit des faits, soit des valeurs. Elle permet en effet l'interprétation et l'intégration de diverses valeurs des sources d'influence pesant sur l'organisation. Cela correspond à des activités telles que la transmission du courrier dans l'organisation ou celle, verbale, d'informations à des subordonnés. Pour le créateur, qui n'a pas ou a très peu de subordonnés, le rôle est d'autant plus réduit. Lorsqu'il est seul, ce rôle est inexistant, mais même lorsqu'il a quelques employés, il est mineur. En effet, dans une petite structure, il n'y a pas de blocage organisationnel: la diffusion de l'information se fait sans difficultés; il n'est pas nécessaire pour le créateur de la formaliser, d'y accorder une attention précise. 
Dans les entreprises nouvellement créées, on observe rarement un circuit formel d'information. Lorsque l'entreprise se développe et que les effectifs augmentent, le créateur doit alors veiller à une bonne circulation interne de l'information, ce qu'il ne fait pas toujours. Le comportement entrepreneurial va parfois de pair avec un autoritarisme certain et la croyance est répandue que moins le personnel en sait, mieux l'entreprise se porte. Cette attitude revient, en fait, à privilégier le pouvoir à court terme au détriment de l'efficacité à moyen terme.

Le cadre comme porte-parole. Ce rôle consiste à transmettre des informations vers l'extérieur de l'organisation, vers son environnement. À cette fin, le cadre participe à des conseils d'administration, traite du courrier et a des contacts concernant la transmission d'information à des personnes extérieures. Qu'en est-il pour le créateur? Sur le plan théorique, ce rôle existe aussi pour le créateur d'entreprise, mais en pratique, il est très réduit. En effet, le créateur d'entreprise est rarement sollicité pour parler au nom de son organisation. Ses contacts avec des organismes professionnels, gouvernementaux ou avec la presse sont assez rares, en tout cas sans commune mesure avec ceux des cadres dirigeants de grandes organisations. Le cadre est souvent amené à agir en tant qu'expert du domaine d'activité de son organisation alors que le créateur est rarement consulté, car il est censé devoir faire ses preuves avant d'être considéré comme un expert. Et on a parfois cette situation paradoxale où un cadre qui était jugé comme un expert et consulté comme tel lorsqu'il travaillait dans une grande organisation redevient un débutant le jour où il crée sa propre entreprise. De nombreux cadres ont d'ailleurs remarqué cette quasi-perte d'existence sociale liée à la création d'entreprise.

\subsection{Les rôles décisionnels}

Ces rôles regroupent toutes les activités du cadre qui sont liées à la prise de décisions importantes concernant l'organisation. Le cadre est impliqué dans ces décisions pour plusieurs raisons : il détient l'autorité formelle et peut donc engager l'organisation dans des actions nouvelles, il dispose des informations nécessaires pour agir ainsi et, enfin, il intègre sous son autorité les décisions stratégiques. La prise de décisions par les managers constitue l'un des aspects de leur travail ayant le plus intéressé les chercheurs. Aussi, de nombreuses publications traitent-elles de ce sujet.

Les rôles décisionnels sont au nombre de quatre: entrepreneur, régulateur, répartiteur de ressources et négociateur.

Le cadre comme entrepreneur. Ce rôle consiste à prendre l'initiative et à assurer la conception de la plupart des changements contrôlés affectant l'organisation. Pour cela, le cadre recherche des occasions favorables dans l'entreprise 
et dans son environnement; il prend l'initiative de projets d'amélioration et supervise la conception de certains projets. Le rôle d'entrepreneur correspond à des activités comme l'examen de la situation, l'élaboration de la stratégie, la conception de projets d'amélioration, c'est-à-dire toutes les activités associées au changement systématique dans l'organisation. Il s'agit d'actions volontaires. C'est un rôle fondamental pour le créateur, et les économistes ne s'y trompent pas en associant le terme d'entrepreneur à la création de nouvelles organisations. Le créateur va tout d'abord, au cours d'une phase d'observation active précédemment décrite, observer son environnement pour y repérer les possibilités d'agir, les occasions d'affaires. Parmi toutes les potentialités, il repère celles qui remplissent deux conditions essentielles : correspondre à son projet stratégique, c'est-à-dire au type d'entreprise qu'il veut créer et développer, et nécessiter pour leur mise en œuvre des moyens matériels, humains et financiers en adéquation avec ses possibilités très souvent limitées. Une fois ce travail préalable effectué, il va devoir agir, transformer cette potentialité en action. Ce passage à l'acte marque la différence entre le créateur véritable et tous ceux dont l'idée de création d'entreprise ne va pas au-delà du projet. Cette attitude entrepreneuriale ne sera pas celle du créateur uniquement lors du démarrage de sa firme, mais tout au long de sa vie professionnelle comme pour les managers étudiés par Mintzberg.

Le cadre comme régulateur. Le rôle de régulateur correspond à des situations involontaires où le cadre doit faire face à des perturbations importantes et inattendues qui affectent l'organisation et trouver rapidement la correction nécessaire. Il s'agit donc des activités permettant de traiter perturbations et crises. Pour un cadre, elles peuvent être de trois types: des conflits entre subordonnés, des conflits entre organisations différentes, et enfin des pertes de ressources ou tout au moins le risque de telles pertes. Cette activité de régulateur est aussi très importante pour le créateur. L'autorégulation d'un système récemment créé est faible et la sensibilité aux perturbations se montre très grande. Une organisation naissante est fragile, une crise sans gravité pour une grande entreprise peut, ici, avoir des conséquences dramatiques. Le créateur doit veiller à ne pas laisser la crise se développer et à mettre en œuvre très rapidement les mesures correctives nécessaires. Cette difficulté peut être encore augmentée par l'inexpérience du créateur débutant. Il se laisse surprendre par les crises et se retrouve incapable de les détecter dès les premières manifestations. Il ne réagit que lorsqu'elles ont atteint une ampleur suffisante pour qu'il s'aperçoive de leur existence. Le cadre confirmé réagit plus vite; dès les premiers symptômes, les mesures qu'il prend sont donc beaucoup plus efficaces. Certains créateurs se lancent dans des activités totalement nouvelles pour eux (situation de mutation); il leur est alors très difficile d'accomplir avec efficacité ce rôle de régulateur. 
Le cadre comme répartiteur de ressources. Ce rôle consiste à assumer la responsabilité de la répartition des diverses ressources organisationnelles. Cette répartition est essentielle, car ces choix, engageant les ressources de l'organisation, contribuent à déterminer la stratégie suivie. Pour le cadre, elle comprend essentiellement trois éléments: l'organisation de son temps de travail, reflet des intérêts de son entreprise et de ses priorités d'action, la programmation du travail de ses subordonnés, et enfin l'octroi d'autorisations. Tout ceci se traduit par des activités comme la gestion de son emploi du temps, la réponse aux demandes d'autorisation, l'affectation des budgets, la programmation du travail des subordonnés... Ce rôle pour le créateur est à la fois important au niveau de ses conséquences et assez simple dans sa mise en œuvre pratique. Il est primordial, car veiller à une utilisation judicieuse de ses ressources est d'autant plus essentiel que celles-ci sont limitées. L'une des principales ressources dont dispose le créateur est son propre temps de travail : il doit savoir établir une distinction entre les tâches déterminantes pour l'avenir de l'entreprise et celles qui le sont moins. Son inexpérience ne doit pas l'amener à gaspiller son temps dans des tâches secondaires, ce qui n'est pas toujours évident. Il est simple dans sa mise en œuvre: l'organisation est de taille réduite et la programmation du travail de quelques subordonnés ne présente pas en général de difficultés majeures. Il en est de même pour l'octroi des autorisations. Sur ce dernier point, il faut ajouter que cela peut constituer pour un créateur débutant l'occasion d'apprendre à dire non. C'est le cas, par exemple, des anciens cadres fonctionnels n'ayant eu aucune responsabilité hiérarchique, ou des jeunes qui créent une entreprise dès la fin de leur cursus scolaire ou universitaire. Autoriser ou refuser, c'est choisir ce qui est important pour la jeune entreprise et doit passer avant le reste.

Le cadre comme négociateur. Le cadre représente son organisation dans les négociations importantes. Pour Mintzberg, il s'agit d'une partie vitale du travail du cadre. En effet, il est symbole de son organisation; sa présence accroît donc la crédibilité des négociations. Il est porte-parole; il représente le système de valeurs et d'information de son organisation. Il est aussi un répartiteur de ressources. En tant que tel, il a l'autorité nécessaire pour engager les ressources de son entreprise. C'est un rôle essentiel pour le créateur qui est partie prenante non seulement aux négociations importantes, mais à toutes celles de son entreprise. Comme il sera vu dans la suite de cette étude, le créateur consacre une part majeure de ses activités à vendre ses produits ou ses services. Or, la négociation est une des composantes principales de l'activité commerciale. Il est également très impliqué dans la fonction production, en particulier, en ce qui concerne les achats. Là encore, il devra mettre en œuvre ses talents de négociateur pour se procurer ce dont il a besoin aux meilleures conditions possibles.

On peut maintenant se demander si la description de Mintzberg est suffisante et s'il ne faut pas rajouter à cette liste un ou plusieurs rôles, spécifiques au 
créateur, qui n'ont pas été décrits dans son étude. Choran (1969) a réalisé une recherche comparable, mais portant sur de petites entreprises. Il estime nécessaire de rajouter deux nouveaux rôles, occupant le huitième du temps des managers observés : celui de spécialiste qui consiste à s'impliquer directement lorsqu'ils jugent «qu'une fonction particulière est vitale pour le bon déroulement des opérations... » et celui d'opérateur remplaçant. La petite entreprise dispose presque toujours de ressources d'appoint insuffisantes et le cadre doit savoir remplacer un salarié absent ou venir compléter des effectifs trop faibles. Pour le créateur, nous ne retiendrons qu'un seul rôle, essentiel, celui d'opérateur. Le créateur qui débute est à la fois dirigeant et exécutant, manager et opérateur. À ce propos, Barbara J. Bird (1986), dans son article «Entrepreneurial behavior: What do entrepreneurs do?» rappelle la spécificité du métier d'entrepreneur: «Les entrepreneurs cependant, ont des rôles uniques dans les organisations qu'ils créent et transforment. Ils sont dirigeants et exécutants.» Au tout début, le créateur constitue bien souvent la seule ressource humaine de son entreprise, il doit donc à la fois exécuter le travail proprement dit, assurer la direction de l'exécution de ce travail et construire l'organisation.

\section{Une variable de contingence: la taille de l'organisation}

L'extrême variété de cadres et du travail qu'ils réalisent a amené Mintzberg (1984) à s'intéresser aux indices de variations entre postes dans les études empiriques et à présenter une théorie contingente de leur travail. Il précise cependant: "Il faut insister sur un point: les études empiriques réalisées jusqu'ici sur le travail du cadre ont mis en évidence plus de similarités que de différences entre les postes.» La cohérence entre cette théorie contingente et les résultats obtenus pour le créateur va donc maintenant être étudiée à partir d'une variable: la taille de l'organisation. Son influence sur le comportement du cadre correspond-elle à une certaine logique lorsqu'on compare cadre et créateur? En d'autres termes, le métier de créateur peut-il être analysé comme une variante du métier de cadre, ou bien au contraire s'agit-il de deux activités professionnelles totalement différentes?

\subsection{Les travaux d'Irving Choran}

Ce chercheur a réalisé une étude comparable sur le plan méthodologique à celle de Mintzberg auprès de trois dirigeants de petites entreprises. Il a retrouvé les six ensembles de caractéristiques du travail et les dix rôles décrits. Des différences toutefois apparaissent entre les dirigeants des grandes et des petites organisations. Ces derniers ont moins d'activités de nature formelle, 
se préoccupent plus du suivi des opérations internes, du travail d'organisation. Ils ont plus d'activités quotidiennes différentes et leur travail est plus marqué par la brièveté et la fragmentation; ils ont davantage recours aux moyens de communication informelle comme le téléphone; ils passent moins de temps en réunions programmées. Leurs contacts externes sont plus restreints et ils passent l'essentiel de leur temps avec leurs subordonnnés, leurs fournisseurs et leurs associés; leur courrier montre des réseaux de communication formelle moins développés. Il existe donc une similitude certaine entre le travail du dirigeant d'une petite organisation et le créateur. Les différences les plus significatives sont liées à l'absence totale de subordonnés ou aux effectifs réduits du créateur: peu de temps consacré aux réunions programmées $(4,84 \%$ contre $21 \%)$ et, par conséquent, plus de temps passé seul au bureau $(47,74 \%$ contre $35 \%)$. Le temps de contact verbal est passé plutôt avec les clients et les fournisseurs $(55,43 \%)$ qu'avec les subordonnés $(9,74 \%)$, contrairement au dirigeant pour lequel ces pourcentages sont respectivement de $38 \%$ et $56 \%$. Le créateur traite encore moins de courrier que le dirigeant (5,1 par jour contre 17) et a une activité très centrée sur le travail de l'organisation. L'étude de la taille de l'entreprise permet donc de dire que le travail du créateur a de nombreux aspects communs avec celui du dirigeant d'une petite organisation et que les différences essentielles sont la conséquence du petit nombre de subordonnés du créateur.

\subsection{Les travaux de Jean-Luc Delpeuch et Anne Lauvergeon}

Répondant aux mêmes préoccupations que Mintzberg, ces deux chercheurs se sont intéressés à la nature concrète des activités des dirigeants et en ont proposé un modèle.

Avant de présenter leurs résultats, il convient d'aborder leur méthodologie puis leur échantillon. Sur le plan méthodologique, les données recueillies l'ont été au cours d'entretiens de deux heures environ avec des cadres dirigeants suivis de rencontres avec leur secrétaire et d'études de leur agenda personnel. Il s'agit donc essentiellement de données indirectes tirées de discours sur des pratiques, d'où les risques de biais plus ou moins importants. L'observation directe, telle que pratiquée par Mintzberg et Hernandez, et permettant la saisie directe et immédiate de données, s'est limitée à une demi-journée avec trois dirigeants. Si cette méthodologie présente des inconvénients, elle offre par contre l'avantage de permettre l'étude d'un échantillon plus important de dirigeants : ici 34. Ces derniers exercent leurs fonctions essentiellement dans de grandes organisations: 27 entreprises ont plus de 500 employés.

La répartition de leurs activités quotidiennes a d'abord été étudiée. Elle présente plusieurs points communs avec les managers observés par Mintzberg. La réunion constitue leur activité essentielle, un dirigeant étant rarement seul: 
«un dirigeant d'entreprise passe 50 à $90 \%$ de son temps de travail en réunions avec un ou plusieurs interlocuteurs». Leur temps de travail a été réparti en trois grandes catégories: travail individuel, réunions et voyages.

Le travail individuel est donc l'activité principale du dirigeant de petite organisation ou du créateur et la réunion, celle du dirigeant d'une grande organisation. Quant aux «déplacements », ils n'ont pas la même signification pour les diverses catégories. Véritables voyages pour les interviewés de Delpeuch et Lauvergeon, il s'agit de simples déplacements de courte durée pour les créateurs observés: visite d'atelier, rendez-vous chez un client potentiel, tournée de chantier, etc. L'étendue du champ d'action de l'organisation influe bien évidemment sur la nature des déplacements de ses dirigeants.

Puis Delpeuch et Lauvergeon ont distingué trois domaines d'actions essentiels dans les activités de dirigeants qualifiés de «sphères» et ont proposé «un modèle des trois sphères » pour décrire leur travail. La première sphère est qualifiée de «statutaire». Il s'agit d'activités programmées à long terme ou à période fixe où la présence du dirigeant s'impose (conseils d'administration, comités d'entreprise, cérémonies diverses, etc.). La seconde est qualifiée de «sphères des remontées ». Elle concerne des activités opérationnelles qui n'ont pas été assumées par les niveaux hiérarchiques inférieurs et remontent jusqu'à la direction, des événements où le fonctionnement habituel de l'organisation est défaillant. Il peut s'agir d'arbitrer un conflit interne ou de prendre une décision délicate et urgente. La troisième est la «sphère de créativité» correspondant à des activités à forte implication personnelle où le dirigeant met sa marque personnelle en fonction de goûts ou de talents particuliers: animation de réunions, contacts internationaux, relations avec les milieux politiques... Cette dernière sphère n'est pas nécessairement disjointe des deux précédentes; elle peut les recouper. Les divers rôles des dirigeants se répartissent entre ces trois sphères.

On les retrouve dans l'activité du créateur qui apparaît là encore analysable comme une variante de travail de dirigeant. La spécificité du travail des créateurs ressort dans leur importance relative. La sphère statutaire regroupe essentiellement des rôles peu importants pour le créateur, comme porte-parole ou figure de rituel. La sphère des remontées ne devient significative qu'au fur et à mesure que l'organisation créée se développe: limitée au tout début de la création, elle va grandissante au bout de quelques mois. Les rôles fondamentaux pour le créateur ressortent bien évidemment de la troisième sphère, celle de la créativité. 


\section{Conclusion}

L'étude réalisée a permis de préciser un certain nombre d'éléments concernant le travail du créateur d'entreprise. Il s'agit d'une activité caractérisée par la brièveté, la variété et la fragmentation; le créateur a une préférence marquée pour l'action et la communication verbale. Enfin, la solitude ressort comme un élément essentiel du métier de créateur. Les rôles essentiels sont ceux d'entrepreneur, de négociateur, de régulateur, d'agent de liaison et d'observateur actif, auxquels il faut ajouter un rôle supplémentaire, celui d'opérateur. Le créateur a, en effet, la particularité d'être à la fois l'exécutant et le dirigeant de son propre travail.

\section{Journée type d'un créateur}

\begin{tabular}{|c|c|c|c|}
\hline Activités & Nombre & Durée moyenne & Durée totale \\
\hline Sessions de travail au bureau & 20,2 & $11 \min 16 \mathrm{~s}$ & $3 \mathrm{~h} 47 \mathrm{~min} 51 \mathrm{~s}$ \\
\hline \multicolumn{4}{|l|}{ Appels téléphoniques } \\
\hline reçus & 3,6 & $4 \min$ & $14 \min 24 \mathrm{~s}$ \\
\hline donnés & 16 & $2 \min 54 \mathrm{~s}$ & $46 \min 24 s$ \\
\hline Réunions programmées & 0,8 & $28 \min 52 \mathrm{~s}$ & $23 \min 05 s$ \\
\hline Réunions non programmées & 9,8 & $8 \min 55 s$ & $1 \mathrm{~h} 27 \min 23 \mathrm{~s}$ \\
\hline Tournées des installations & 6,5 & $11 \min 59 \mathrm{~s}$ & $1 \mathrm{~h} 17 \mathrm{~min} 53 \mathrm{~s}$ \\
\hline TOTAL & 56,9 & $8 \min 23 s$ & 7 h 57 min \\
\hline
\end{tabular}

Sur le plan méthodologique, cette recherche relève de l'observation directe, une voie jusqu'à ce jour peu suivie pour étudier l'entrepreneuriat. Si la présence d'un chercheur dans une grande organisation est aujourd'hui assez bien admise et pose un nombre limité de problèmes pratiques, il n'en est pas de même dans le cas d'une entreprise en cours de création. Le créateur est seul, face à de multiples problèmes, et la présence d'un chercheur à ses côtés constitue pour lui une contrainte supplémentaire à un moment de sa carrière professionnelle où il a d'autres préoccupations plus urgentes que de faciliter la recherche en sciences de gestion. Cette méthode présente pourtant un grand intérêt. La présence sur le terrain permet le recueil de données beaucoup plus riches que l'enquête par questionnaire (les anecdoctes). Autant de matériel pouvant servir de base à l'élaboration de théories. Ces deux méthodes pour recueillir des données se complètent l'une l'autre, et les obstacles à l'observation directe ne doivent pas faire renoncer le chercheur. Mintzberg (1979) relève lui aussi l'intérêt de la pratique du terrain et la nécessité de disposer à la fois de données «systématiques» et «anecdotiques» pour progresser dans la connaissance de son objet de recherche: 
Car, alors que les données systématiques forment la base de nos théories, ce sont les données anecdotiques qui nous permettent « d'ériger le bâtiment ». La construction de la théorie semble requérir une description riche, la richesse qui vient de l'anecdote. Nous découvrons de nombreuses sortes de relations dans nos données « hard », mais ce n'est que par l'utilisation de ces données «soft » que nous pouvons les expliquer et l'explication est évidemment le but de la recherche. Je crois que le chercheur qui ne va jamais sur le terrain, qui récolte des données quantitatives à distance sans anecdotes pour les étayer, aura toujours des difficultés à expliquer des relations intéressantes (bien qu'il puisse les découvrir).

Le chercheur doit également garder constamment à l'esprit la nécessité de se recentrer sur son étude, et la relation de sympathie qui s'établit parfois avec le créateur ne doit pas atténuer ses capacités de recul, de lecture et d'analyse.

\section{Bibliographie}

BIRD, B.J. (1986), «Entrepreneurial behavior: What do entrepreneurs do ?» Frontiers of Entrepreneurship Research, p. 168-170.

ChORAN, I. (1969), «The manager of a small company », Montréal, Université McGill, Mémoire de maîtrise non publié.

DelPeuch, J.L. et A. LAUVergeon (1986), «Sur les traces des dirigeants », Annales des Mines, Série Gérer et Comprendre, n² 2, p. 20-31.

GARTNER, W.B. (1989), «Who is an Entrepreneur? Is the wrong question», Entrepreneurship Theory and Practice, vol. 13, $\mathrm{n}^{\circ} 4$, p. 47-67.

MINTZBERG, H. (1979), «An emerging strategy of " direct" research», Administrative Science Quarterly, vol. 24, ${ }^{\circ} 4$, p. 582-589.

MintzBeRG, H. (1984), Le manager au quotidien, Paris, Les Éditions d'Organisation.

SARBIN, T.R. et V.L. AlLEN (1968), «Role theory », dans G. Lindzey et E. Aronson, The Handbook of Social Psychology, Reading (Mass.), Addison-Wesley, vol. 1, p. 488-567. 